\title{
Abnormality Detection of TB using Instance Learned Classifier on Lung CT Images
}

\author{
P. Prasanna Kumari, B. Prabhakara Rao
}

\begin{abstract}
Tuberculosis is one of the serious health threatening disease which is more ubiquitous in African countries. According to World Health Organization[1], it is the second leading cause of death from an infection after HIV and 10.4 million cases of TB and 1.3 million deaths were reported worldwide in 2017 based on the estimates of Centre for Disease Control and Prevention $(C D C)$. This paper presents abnormality detection of $T B$ using an instance based learning classifier known as the KNN classifier an SVM classifier. If input image classified as abnormal then abnormal region is extracted using the segmentation. The system has been tested on the number of lung CT scan images.
\end{abstract}

Keywords Support Vector Machine (SVM), K-Nearest Neighbor (KNN), Computed Tomography (CT), Tuberculosis $(\mathrm{TB})$.

\section{INTRODUCTION}

Tuberculosis is major infectious disease caused by a mycobacterium tuberculosis. It affects the lungs and attacks the low immune bodies through air by coughing and sneezing. Unfortunately diagnosing TB is still a major challenge. Medical images are obtained from several modalities like X-ray, MRI, Computed Tomography(CT), Ultrasound, PET etc. The more reliable modality for the detection of TB is computed tomography. Computed Tomography images can be distinguished for dissimilar tissues according to their gray level. Lot of research work done to assist TB diagnosis in the area of medical image processing and several Automatic diagnosis systems are developed. Computationally and conceptually the simple technique that provides better accuracy is the K-Nearest Neighbor (K-NN) classifier. The k-NN algorithm is based on voting function and a distance function in $\mathrm{k}$-Nearest Neighbors, the popular measure of distance considered is the Euclidean distance. Another important classifier is SVM. In this paper classification done for both KNN an SVM classifiers and compared their performance measures.

\section{RELATED WORK}

Jaime Melendez, et al, [3] proposed CAD based multiple -instance learning approach for detection of TB using chest X-Rays. Stefan Jegar,et al,[4] presented a system for automatic detection of tuberculosis using the Japanese Society of Radiological Technology (JSRT) data set. Log Gabor filters based on Log Gabor Wavelets are used due to

Revised Version Manuscript Received on 16 September, 2019.

P.PrasannaKumari, Research Scholar, ECE department, JNTUK, Kakinada, A.P, India.

(Email: prasannasudhirg@gmail.com)

Dr.B.PrabhakaraRao, Program Director, School of Nanotechnology, IST, JNTUK, Kakinada, A.P, India.

(Email: drbprjntuk@gmail.com) limited bandwidth. Elveren and Yumusak [5] used multilayer neural network obtained success rate of $94.8 \%$ by using genetic algorithm for the detection of TB, Er et al [6] used artificial neural network and achieved 94.88 success rate in their work. Dongardive et al.[7] achieved 93\% success rate in diagnostic accuracy by using identification tree (IDT)

\section{MATERIALS AND METHODS USED}

Data set consisting of 55 CT images, in which $50 \%$ images are applied for Training process and remaining $50 \%$ applied for Testing process.
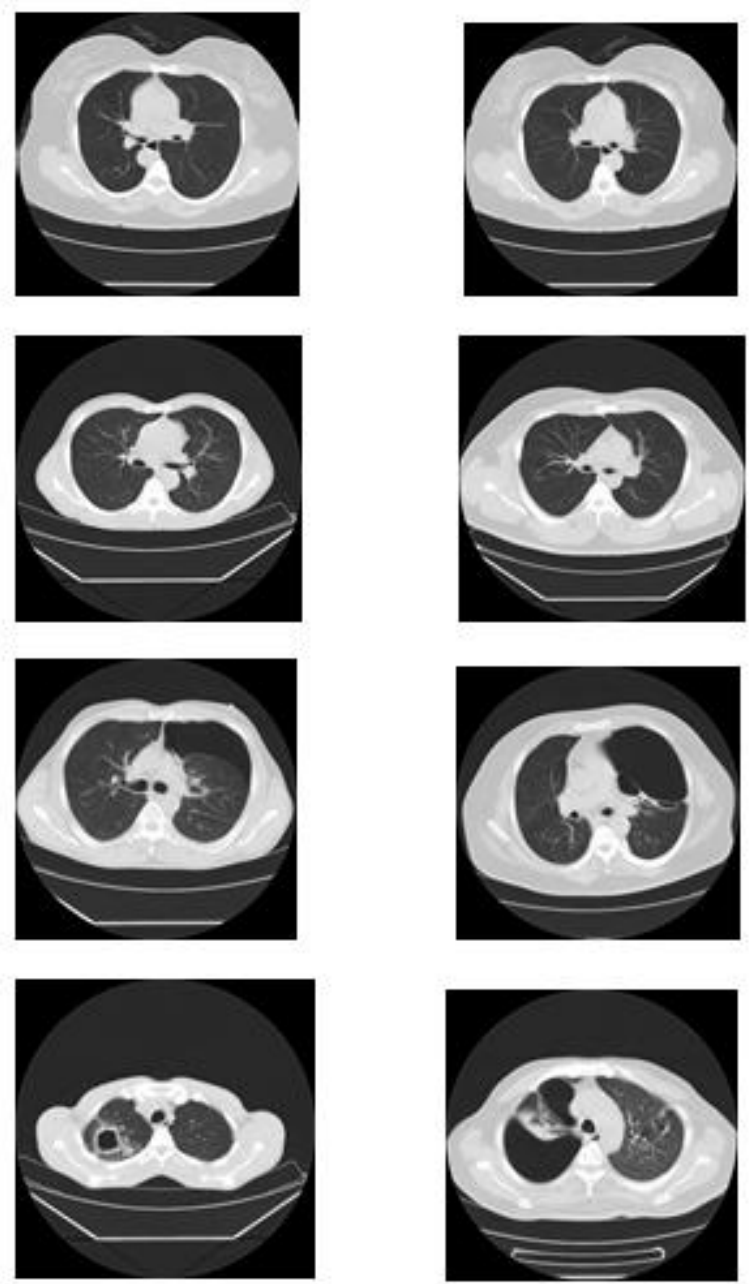

Fig 3.1 Sample Input Images in the data set Methodology

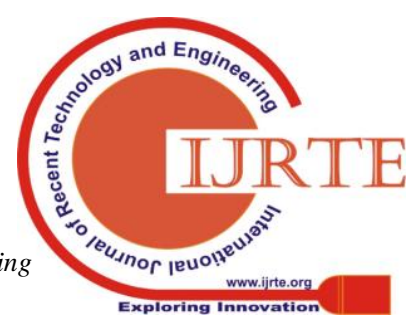


In the proposed work input is CT lung images for which preprocessing is done to enhance the image into suitable form by converting input image into gray scale image,

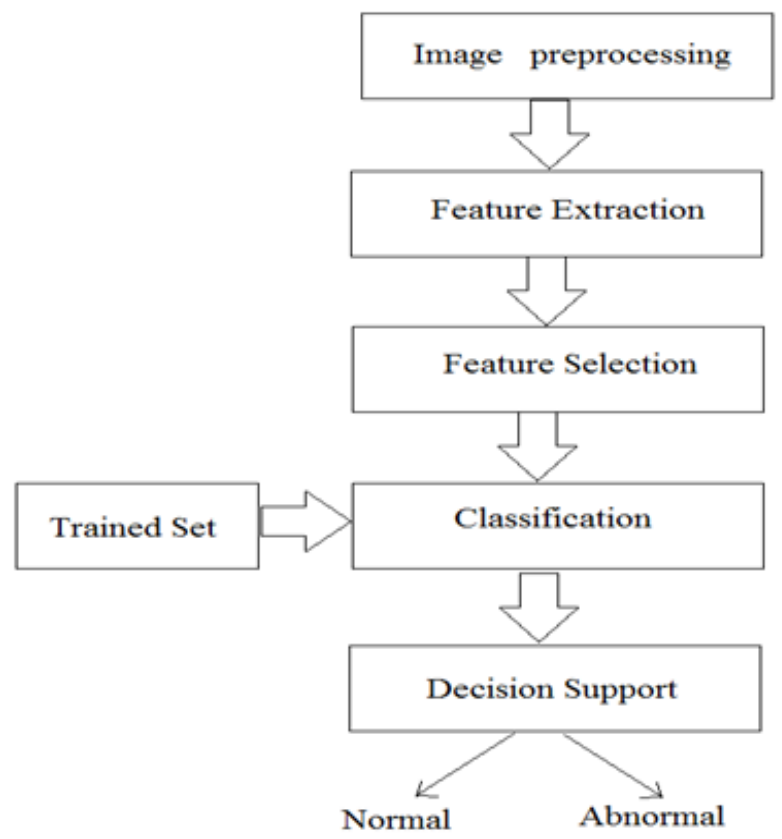

Fig 3.2 Flowchart representation of proposed method

Extracted the set of features and reduced the features set using roughest theory based feature selection method and the classification of the images is done through the SVM classifier and compare the results with KNN classifier by using MATLAB . In the phase of Classification, we used two classifiers namely K- Nearest Neighbor classifier and SVM Classifier to classify whether test input image is Abnormal or Normal Class.

\section{K-Nearest Neighbor classifier:}

KNN classifier has several advantages like easy implementation, less computational complexity and less time taken for classification. Similarity between instances can be identified by means of k-nearest neighbors. If majority represents abnormal pixels then it is classified as abnormal otherwise it is classified as normal

\section{Support Vector Machine classifier:}

SVM is associate correct learning technique. . A support vector machine constructs associate best hyper plane or set of hyper planes in a very high dimensional area, which may be used for classification and regression. It achieves sensible separation by choosing hyper plane that has largest distance to the closest information points of a category as.

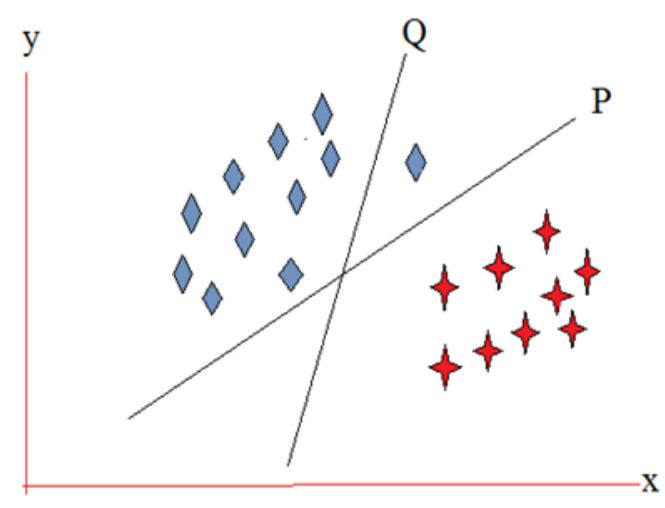

Fig 3.3. SVM separating hyper planes

\section{RESULTS AND DISCUSSIONS}

The proposed system was tested on dataset consisting of $55 \mathrm{CT}$ images that comprised of both normal and abnormal images. Some images in the dataset are used for training, remaining images are used for testing purpose.

\section{Performance Measures}

From a confusion matrix shown in table I, we will live performance measures of a classifier like Positive prophetic worth, Negative prophetic worth, Accuracy, Sensitivity, Specificity, False Negative Rate, False Positive Rate and False Discovery Rate.

Table I. confusion Matrix

\begin{tabular}{|c|l|l|l|}
\hline $\begin{array}{c}\text { Confusion } \\
\text { matrix }\end{array}$ & \multicolumn{2}{|c|}{ Predicted label } \\
\hline & & Positive & Negative \\
\hline \multirow{3}{*}{$\begin{array}{l}\text { Known } \\
\text { Label }\end{array}$} & Positive & $\begin{array}{l}\text { True } \\
\text { Positive } \\
\text { (TP) }\end{array}$ & $\begin{array}{l}\text { False } \\
\text { Negative } \\
\text { (FN) }\end{array}$ \\
\cline { 2 - 4 } & Negative & $\begin{array}{l}\text { False } \\
\text { Positive } \\
\text { (FP) }\end{array}$ & $\begin{array}{l}\text { True } \\
\text { Negative } \\
\text { (TN) }\end{array}$ \\
\hline
\end{tabular}

Table.I represents a confusion matrix for binary classification. Where TP estimates malignant as malignant.False Negative estimates malignant as benign [8]. True Negative estimates benign as benign, False Positive predicts benign as malignant. The performance of the both support vector machine and $\mathrm{k}$ nearest neighbor classifiers are compared by calculating the Accuracy, Sensitivity, Specificity, False Positive Rate(FPR), False Discovery Rate (FDR) and False Negative Rate (FNR). 
i) Positive Predictive value

$P P V=\frac{\text { Number of True Positives }}{\text { Number of True Positive }+ \text { Number of False positives }}$

ii) Negative Predictive Value
The Support Vector Machine (SVM) Classifier achieves maximum accuracy of $82.5 \%$, sensitivity of $80 \%$ and specificity of $83.3 \%$ as shown in the Table II. The Area Under Curve (AUC) is a measure of how well a parameter can distinguish between normal or abnormal images. AUC can be obtained from the Receiver Characteristic (ROC) Curve plotted between True positive rate and false positive rate

$$
N P V=\frac{\text { Number of True Negatives }}{\text { Number of True Negatives }+ \text { Number of False Negatives }}
$$

Iii) Accuracy $=\frac{T P+T N}{T P+T N+F P+F N}$

iv) Sensitivity $=\frac{\mathrm{TP}}{\mathrm{TP}+\mathrm{FN}}$

v) Specificity $=\frac{\mathrm{TN}}{\mathrm{TN}+\mathrm{FP}}$

vi) False Negative Rate $F N R=\frac{\mathrm{FN}}{\mathrm{TP}+\mathrm{FN}}$

vii) False Positive Rate $F P R=1-\frac{T N}{T N+F P}$

viii) False discovery Rate $F D R$

$$
=\frac{\text { Number of False Positives }}{\text { Number of True Positives }+ \text { Number of False positives }}
$$

\begin{tabular}{|c|c|c|c|c|c|c|c|c|c|c|c|c|c|c|}
\hline $\begin{array}{l}\text { Images } \\
\text { used for } \\
\text { Training }\end{array}$ & $\begin{array}{l}\text { Images } \\
\text { used } \\
\text { for } \\
\text { Testing }\end{array}$ & Method & TP & TN & FP & $\mathrm{FN}$ & Sensitivity & Specificity & Accuracy & PPV & NPV & FPR & FNR & FDR \\
\hline \multirow[b]{2}{*}{$50 \%$} & \multirow[b]{2}{*}{$50 \%$} & SVM & 11 & 10 & 2 & 4 & $73.33 \%$ & $83.33 \%$ & $77.78 \%$ & $84.62 \%$ & $71.43 \%$ & $16.67 \%$ & $26.67 \%$ & $15.38 \%$ \\
\hline & & KNN & 4 & 7 & 5 & 11 & $26.67 \%$ & $58.33 \%$ & $40.74 \%$ & $44.44 \%$ & $38.89 \%$ & $41.67 \%$ & $73.33 \%$ & $55.56 \%$ \\
\hline \multirow[b]{2}{*}{$70 \%$} & \multirow[b]{2}{*}{$30 \%$} & SVM & 4 & 10 & 2 & 1 & $80.00 \%$ & $83.33 \%$ & $82.35 \%$ & $66.67 \%$ & $90.91 \%$ & $16.67 \%$ & $20.00 \%$ & $33.33 \%$ \\
\hline & & $\mathrm{KNN}$ & 3 & 7 & 5 & 2 & $60.00 \%$ & $58.33 \%$ & $58.82 \%$ & $37.50 \%$ & $77.78 \%$ & $41.67 \%$ & $40.00 \%$ & $62.50 \%$ \\
\hline \multirow[b]{2}{*}{$75 \%$} & \multirow[b]{2}{*}{$25 \%$} & SVM & 1 & 10 & 2 & 1 & $50.00 \%$ & $83.33 \%$ & $78.57 \%$ & $33.33 \%$ & $90.91 \%$ & $16.67 \%$ & $50.00 \%$ & $66.67 \%$ \\
\hline & & $\mathrm{KNN}$ & 1 & 7 & 5 & 1 & $50.00 \%$ & $58.33 \%$ & $57.14 \%$ & $16.67 \%$ & $87.50 \%$ & $41.67 \%$ & $50.00 \%$ & $83.33 \%$ \\
\hline
\end{tabular}

Table II . Performance Comparison of SVM and KNN classifiers for TB diagnosis 

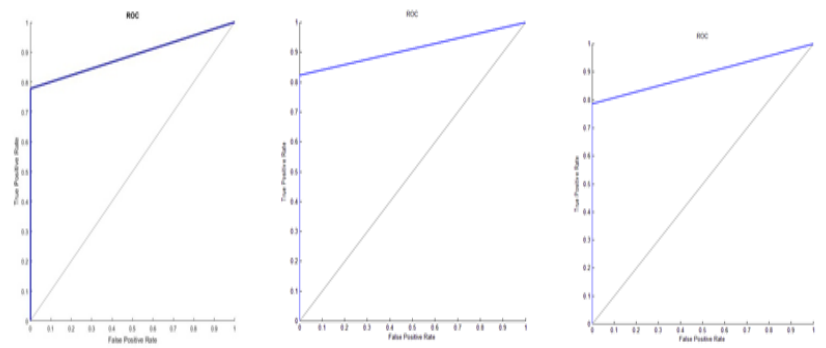

Fig 4.1. ROC curves for SVM Classifier
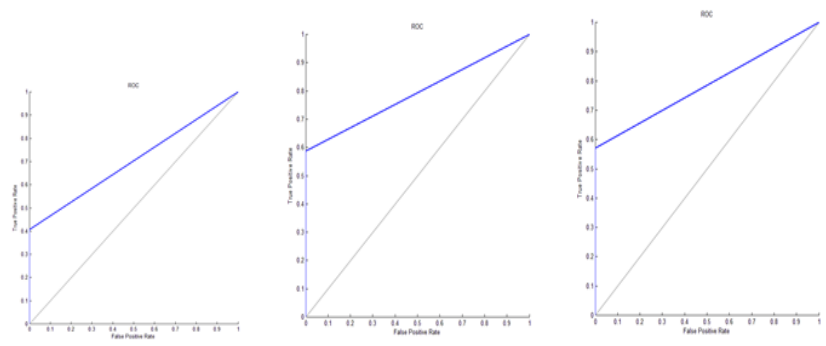

Fig 4.2 ROC curves for KNN classifier

After the process of classification, if the input lung CT image is identifies as abnormal then abnormal region is extracted by using the process called segmentation. Segmentation is done with the help of morphological operations like dilation and erosion. The resultant images are as shown in the Fig. 4.3.
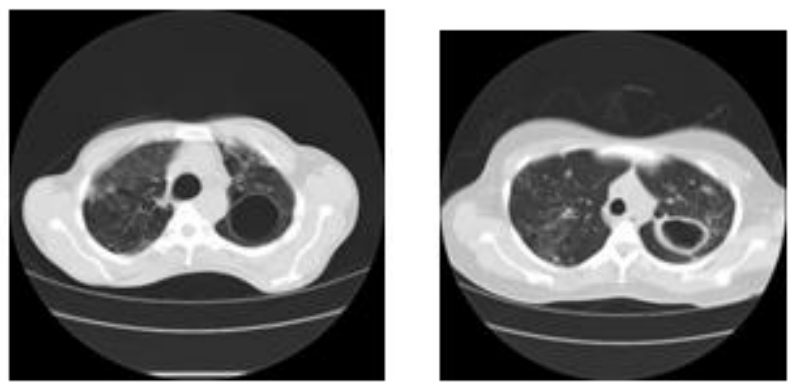

(a)
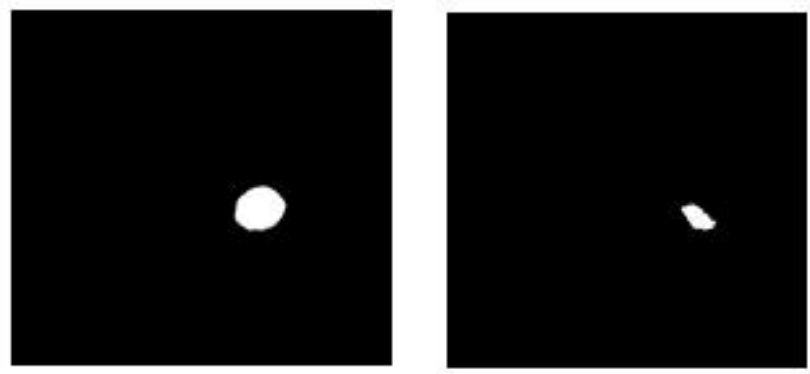

(b)

Fig 4.3 (a) Abnormal Images

(b) Segmented Images

\section{CONCLUSION}

In this paper we have used two different classifiers and identified the best one in detecting Tuberculosis. By taking lung Computed Tomography as input and measured the accuracy, sensitivity, specificity, FPR,FNR and FDR of both KNN and SVM classifiers. SVM classifier was proved as the best in achieving more accuracy from the results.

\section{REFERENCES}

1. World Health Org.,Global T.B. report 2017

2. Bo QIU,Chang Sheng XU,Qi TIAN,' associate degree Automatic organisation Applied In Medical Images", IEEE, ICME, 2006,p1045-1048

3. Jaime Melendez, et al, "A novel multiple-instance learning-based approach to computer-aided detection of T.B. on chest $x$-Rays ", IEEE Transactions on Medical Imaging, vol. 34, no. 1, January 2015, pp.179-192.

4. S. Jaeger, A. Karargyris, S. Antani, and G. Thoma, "Detecting T.B. in radiographs victimisation combined respiratory organ masks", International Conference IEEE Engineering in medication and Biology Society (EMBS), 2012, pp. 4978-4981.

5. Elveren E, Yumusak N. sickness \} disease designation victimisation artificial neural network trained with genetic rule. J Medical Syst 2011; 35: 329.

6. Er O, Temurtas F, Tanrikulu AC. T.B. malady designation victimisation Artificial Neural Networks. J Master of Education Syst 2010; 34: 299

7. DongardiveJ, Xavier A, Jain K, Abraham S. Classification and Rule-Based Approach to Diagnose T.B..AdvComput and Commun 2011; 328.

8. S. Ashwin, J. Ramesh, S. Aravind Kumar, K. Gunavathi. "Efficient and reliable respiratory organ nodule detection employing a neural network primarily based pc assisted designation system", 2012.

\section{AUTHOR'S BIOGRAPHIES}

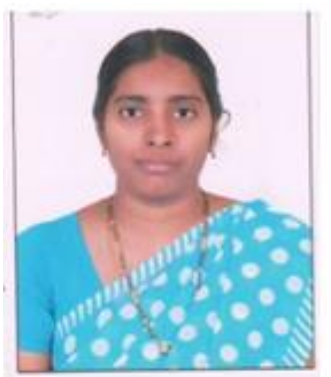

Mrs. P.PrasannaKumari received her B. Tech degree in Electronics and Communication Engineering from Jawaharlal Nehru Technological University, Kakinada, India in 2003 and M.Tech from Jawaharlal Nehru Technological University, Kakinada, India in 2010. She is pursing $\mathrm{PhD}$ in JNTU ,Kakinada India. Her areas of interest include Image Processing ,wireless networks and Embedded systems. Currently She is working in Pragati Engineering college, Surampalem,Andhrapradesh state, India

Dr. B. PrabhakaraRao received his B.Tech degree in ECE from Sri Venkateswara University, India in 1979 and M.Tech from Sri Venkateswara University, India in 1982. He obtained his $\mathrm{PhD}$ degree from Indian Institute of Science, India in 1995. He is currently working as a Program Director of

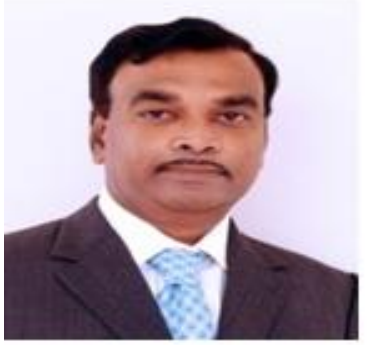
IST, JNTUK, Kakinada, India He published several papers in national journals, international journals and presented papers at conferences. His areas of interest include optical communications, Microwave Engineering, satellite communications, cellular and mobile communications. He is Fellow of IETE and IE (I). He is life member of ISTE 